\title{
A New Test of the Einstein Equivalence Principle and the Isotropy of Space
}

\author{
Mark P. Haugan and Thierry F. Kauffmann \\ Department of Physics, Purdue University 1396, West Lafayette IN 47907
}

()

\begin{abstract}
Recent research has established that nonsymmetric gravitation theories like Moffat's NGT predict that a gravitational field singles out an orthogonal pair of polarization states of light that propagate with different phase velocities. We show that a much wider class of nonmetric theories encompassed by the $\chi g$ formalism predict such violations of the Einstein equivalence principle. This gravity-induced birefringence of space implies that propagation through a gravitational field can alter the polarization of light. We use data from polarization measurements of extragalactic sources to constrain birefringence induced by the field of the Galaxy. Our new constraint is $10^{8}$ times sharper than previous ones.
\end{abstract}

04.80.+z, 04.50.+h, 96.60.Tf

Typeset using REVTEX 


\section{INTRODUCTION}

Gabriel [1] et al. recently established that members of a certain class of nonmetric gravitation theories predict that space is anisotropic and birefringent. These nonsymmetric theories, for which Moffat's NGT [2] is the prototype, have been studied extensively as potentially viable alternatives to general relativity, but they predict that a gravitational field singles out an orthogonal pair of linear polarization states of light which propagate through the field with different phase velocities. The difference in phase velocities could, in principle, be measured in local test experiments [回] and, so, violates the Einstein equivalence principle. In practice, the sharpest constraints on the magnitude of such gravity-induced birefringence will be inferred from limits on the cumulative effect that propagation through a gravitational field has on the polarization of light. Gabriel [3] et al. consider the effect that the Sun's field could induce and use measurements of the polarization of solar spectral lines to impose sharp new constraints on nonsymmetric gravitation theories. Krisher [4] considers the effect the Galaxy's field could induce and uses pulsar polarization observations to impose a complementary constraint on NGT.

In this paper we show that the class of nonmetric gravitation theories which predict spatial anisotropy or birefringence is far more extensive than the class of nonsymmetric theories. Indeed, one must take pains to define a nonmetric coupling between the gravitational and electromagnetic fields that does not induce anisotropy and birefringence. Consequently, searching for effects of propagation through a gravitational field on the polarization of light provides tests of the Einstein equivalence principle that have a power, generality and significance comparable to those of more familiar atomic anisotropy (Hughes-Drever) tests [5] and Eötvös and gravitational redshift tests [6]. Recent theoretical developments provide a strong motivation for precise new tests of the Einstein equivalence principle. For example, interest in scalar-tensor and multi-tensor gravitation theories has been rekindled by the proliferation of scalar and tensor fields in effective field theories derived from string physics and several threads of quantum gravity research suggest that connection rather than metric may be 
fundamental.

The view that searching for evidence of gravity-induced birefringence tests the Einstein equivalence principle is not unprecedented. Ten years ago Ni [7] remarked that nonmetric gravitation theories encompassed by his $\chi g$ formalism could predict birefringence of the type we study. He also noted that pulsar polarization observations could constrain this possibility. Perhaps the significance of these comments was overlooked at the time because no gravitation theories predicting such birefringence were known and because the effective time resolution of pulsar polarization observations is limited. In any event, their significance is now clear. Theories like NGT do predict gravity-induced birefringence and we use galaxy polarization observations to constrain this possibility far more stringently than Ni or Krisher were able to using pulsar polarization observations. Indeed, the linear and circular polarization data for galaxies that we analyze have an effective time resolution that is more than $10^{8}$ times that of Krisher's pulsar polarization data.

\section{ELECTRODYNAMICS IN A BACKGROUND GRAVITATIONAL FIELD}

A theory of gravity does two things. It specifies a free, though generally nonlinear, dynamics of the gravitational field and it specifies the coupling between the gravitational field and matter. The latter determines both how matter generates and how it responds to gravity.

In the case of general relativity these conceptually distinct components of gravitation physics are discernible in the standard form of the Einstein field equation,

$$
G_{\mu \nu}=8 \pi T_{\mu \nu}
$$

The structure of the Einstein tensor, on the left-hand side, specifies the general relativistic dynamics of the gravitational field itself. The form of the stress-energy tensor, on the righthand side, specifies the way in which matter acts as a source of gravity.

These same aspects of general relativity and other Lagrangian-based theories of gravity 
are even more clearly discernible in the structure of the action principles from which their field equations follow,

$$
\delta \int\left(\mathcal{L}_{G}+\mathcal{L}_{N G}\right) d^{4} x=0
$$

The gravitational part of the Lagrangian density, $\mathcal{L}_{G}$, is distinguished by the fact that it depends only on gravitational fields. Its variational derivative with respect to these fields defines their dynamics. The variational derivative with respect to the metric of general relativity's gravitational Lagrangian density, $\sqrt{-g} R / 16 \pi$, is $\sqrt{-g} G_{\mu \nu} / 16 \pi$. The corresponding derivative of its nongravitational Lagrangian density is $\sqrt{-g} T_{\mu \nu} / 2$ and, so, the form of $\mathcal{L}_{N G}$, which depends on both matter and gravitational fields, specifies the way in which matter generates gravity. Its reciprocal role of specifying the way in which matter responds to a gravitational field follows from the action principle (2) on variation with respect to matter fields rather than gravitational ones. This yields the matter equations of motion. In general, these need not follow directly from the gravitational field equations as they do in general relativity.

Experimental tests of the Einstein equivalence principle focus on the behavior of matter responding to a background gravitational field. Their significance lies in the way they constrain the form of $\mathcal{L}_{N G}$ and, thus, provide an empirical foundation for one of gravitation physics' fundamental components. Tests that reveal no violation of the Einstein equivalence principle force the form of the nongravitational Lagrangian density toward ones that admit a metric representation, a representation in which a single metric tensor field couples universally [8] to all matter.

The experimental tests of the Einstein equivalence principle that we analyze in this paper involve the propagation of polarized light through a background gravitational field. We are, therefore, concerned with only that part of the nongravitational Lagrangian density which governs the dynamics of the electromagnetic field and we base our analysis on a general model of this Lagrangian density introduced by $\mathrm{Ni}$ [9]. One advantage of this approach is that the results of our analysis are easily specialized to yield the predictions of any theory 
of gravity whose structure is encompassed by Ni's $\chi g$ formalism. The other advantage is that a broad consideration of conceivable nonmetric couplings provides a context in which to judge how different experimental tests of the Einstein equivalence principle compete with and complement one another and, so, to clarify the strengths and weaknesses of the empirical foundation of one of the most fundamental aspects of gravitation physics.

The structure of the $\chi g$ formalism is consistent with the basic tenets of the Dicke framework [6]. Spacetime is a differentiable manifold and matter fields and phenomenological gravitational potentials are tensor fields. Given the apparent smoothness of space and time and the view that physics should not depend in an essential way on a choice of coordinates, it is difficult to fault these axioms as a basis for phenomenology. Furthermore, it is assumed that as gravity is "turned off" the nongravitational Lagrangian density, $\mathcal{L}_{N G}$, reduces smoothly to the corresponding special relativistic Lagrangian density. The most general Lagrangian density consistent with these axioms and with assumptions of electromagnetic gauge invariance, linearity of the electromagnetic field equations and the absence of couplings to derivatives of gravitational potentials is

$$
\mathcal{L}_{E M}=-\frac{1}{16 \pi} \chi^{\alpha \beta \gamma \delta} F_{\alpha \beta} F_{\gamma \delta}
$$

where the electromagnetic field is related to a 4-potential in the usual way, $F_{\alpha \beta}=A_{\beta, \alpha}-A_{\alpha, \beta}$ and where $\chi^{\alpha \beta \gamma \delta}$ is a tensor density that provides a phenomenological representation of the gravitational field. Note the analogy between $\chi^{\alpha \beta \gamma \delta}$ and a macroscopic electrodynamic constitutive tensor density.

To see how the phenomenological field $\chi^{\alpha \beta \gamma \delta}$ is constructed from a specific theory's scalar, vector and tensor gravitational fields, the structure of the theory's electromagnetic Lagrangian density can be matched to that of $\mathcal{L}_{E M}$ in Eq. (3). In metric theories of gravity it is constructed from the metric tensor alone, $\chi^{\alpha \beta \gamma \delta}=\frac{1}{2} \sqrt{-g}\left(g^{\alpha \gamma} g^{\beta \delta}-g^{\alpha \delta} g^{\beta \gamma}\right)$.

Notice that $\chi^{\alpha \beta \gamma \delta}$ inherits symmetries analogous to those of the Riemann tensor from the antisymmetry of $F_{\alpha \beta}$ and from the symmetrical way in which it appears in Eq. (3). Consequently, $\chi^{\alpha \beta \gamma \delta}$ has twenty-one independent components. Ni says that his formalism 
has twenty-one nonmetric degrees of freedom because, in general, one cannot exploit the formalism's covariance to transform away any of these independent component potentials. The freedom to redefine coordinates is exhausted by putting the formalism's $g_{\alpha \beta}$ field into a standard form. This field represents the coupling between the gravitational field and scalar particles.

\section{GEOMETRIC OPTICS IN A BACKGROUND GRAVITATIONAL FIELD}

In this paper we consider the propagation of light through relatively weak gravitational fields that vary on length and time scales that are far longer than the light's wavelength and period respectively. We, therefore, adapt the coordinate system in which the Lagrangian density (3) is represented to the weak-field limit and we employ the methodology of geometric optics to analyze the light's propagation.

In a Lorentzian coordinate system the electromagnetic Lagrangian density of special relativity has the form in Eq. (3) with $\chi^{\alpha \beta \gamma \delta} \equiv \frac{1}{2}\left(\eta^{\alpha \gamma} \eta^{\beta \delta}-\eta^{\alpha \delta} \eta^{\beta \gamma}\right)$. Consequently, in the weak field limit we can find quasi-Lorentzian coordinate systems in which the field $\chi^{\alpha \beta \gamma \delta}$ has the form

$$
\chi^{\alpha \beta \gamma \delta} \equiv \frac{1}{2}\left(\eta^{\alpha \gamma} \eta^{\beta \delta}-\eta^{\alpha \delta} \eta^{\beta \gamma}\right)+\delta \chi^{\alpha \beta \gamma \delta}
$$

with $\delta \chi^{\alpha \beta \gamma \delta} \ll 1$. Such a decomposition of $\chi^{\alpha \beta \gamma \delta}$ is covariant with respect to Poincaré transformations provided that boost velocities too close to unity are not allowed. Note that this is a purely formal covariance. The background gravitational field may well single out a preferred frame [6].

Geometric optics treats the propagation of locally plane electromagnetic waves. The amplitude and phase representation of such a wave,

$$
\mathbf{E}=\mathbf{A}_{E} e^{i \Phi}, \quad \mathbf{B}=\mathbf{A}_{B} e^{i \Phi} .
$$

is characterized by the fact that derivatives of the vector amplitudes $\mathbf{A}_{E}$ and $\mathbf{A}_{B}$ are small compared to derivatives of the rapidly varying phase function $\Phi$. Since we are interested 
in the propagation of high-frequency electromagnetic radiation through a weak background gravitational field that varies slowly in space and time, derivatives of the background field are also small compared to derivatives of $\Phi$. We base the analysis that follows on the eikonal equation which determines local coordinate velocities of wave propagation. This equation is derived by inserting the representation (5) into the electromagnetic field equations and ignoring all derivatives other than those of the phase function. Propagation equations for the vector amplitudes $\mathbf{A}_{E}$ and $\mathbf{A}_{B}$ and equations governing post-geometric-optic corrections can be derived by taking systematic account of smaller derivatives but we shall not need these equations here.

Neglecting derivatives of the slowly varying background field, the electromagnetic field equation that follows from Eq. (3) is

$$
\chi^{\alpha \beta \gamma \delta} F_{\gamma \delta, \beta}=0
$$

Defining electric and magnetic fields via $F_{i 0} \equiv E_{i}$ and $F_{j k} \equiv \epsilon_{j k l} B_{l}$ and employing the decomposition (4) of $\chi^{\alpha \beta \gamma \delta}$, this can be written somewhat more transparently as

$$
\nabla \cdot \mathbf{E}+\text { terms proportional to } \delta \chi \text { and } \mathbf{E} \text { or } \mathbf{B}=0
$$

and

$$
\nabla \times \mathbf{B}-\frac{\partial \mathbf{E}}{\partial t}+\text { terms proportional to } \delta \chi \text { and } \mathbf{E} \text { or } \mathbf{B}=0
$$

The electric and magnetic fields also satisfy

$$
\nabla \times \mathbf{E}+\frac{\partial \mathbf{B}}{\partial t}=0
$$

and

$$
\nabla \cdot \mathbf{B}=0
$$

by virtue of the fact that $F_{\alpha \beta}$ and, so, $\mathbf{E}$ and $\mathbf{B}$ are derived from potentials in the usual way.

To derive the eikonal equation we insert the representation (5) of a locally plane wave into the field equations (7) - (10) and neglect all derivatives other than those of the rapidly varying 
phase function $\Phi$. Let $k_{\mu}$ denote the gradient of this function, $k_{\mu} \equiv \partial_{\mu} \Phi \equiv(\partial \Phi / \partial t, \nabla \Phi) \equiv$ $(-\omega, \mathbf{k})$, so that Eqs. (9) and (10) become

$$
\mathbf{A}_{B}=\frac{\mathbf{k} \times \mathbf{A}_{E}}{\omega}
$$

and

$$
\mathbf{k} \cdot \mathbf{A}_{B}=0 .
$$

The latter of these implies that the magnetic field of a locally plane wave is transverse to the direction in which the wave propagates. Since Eq. (7) becomes

$$
\mathbf{k} \cdot \mathbf{A}_{E}=\text { terms proportional to } \delta \chi \text { and } \mathbf{A}_{E} \text { or } \mathbf{A}_{B} \text {, }
$$

the electric field is guaranteed to be purely transverse only in the absence of gravity. To first

order in the small $\delta \chi^{\alpha \beta \gamma \delta}$ that represent the weak background gravitational field, Eq. (13) expresses a wave's longitudinal electric field component in terms of its dominant transverse field components. To this same order, $O(\delta \chi)$, Eqs. (11) and (13) imply

$$
\mathbf{A}_{E}=-\frac{\omega}{k^{2}} \mathbf{k} \times \mathbf{A}_{B}+\text { terms proportional to } \delta \chi \text { and } \mathbf{A}_{B},
$$

which expresses $\mathbf{A}_{E}$ in terms of the two independent components of $\mathbf{A}_{B}$, and Eqs. (8), (11) and (14) imply the eikonal equation

$$
\left(1-\frac{\omega^{2}}{k^{2}}\right) \mathbf{A}_{B}=\text { terms proportional to } \delta \chi \text { and } \mathbf{A}_{B} .
$$

Since the magnetic amplitude $\mathbf{A}_{B}$ has two independent components and since $\omega / k$ is coordinate phase velocity, finding the two independent polarization states that propagate with well-defined phase velocities is a matter of solving a two-dimensional eigenvalue problem. Note that in the absence of gravity the right-hand side of Eq. (15) vanishes implying, as one expects, $\omega / k=1$ regardless of polarization.

Before examining the explicit form of Eq. (15) it is convenient to make a $3+1$ decomposition of $\delta \chi^{\alpha \beta \gamma \delta}$ into a set of $\mathrm{SO}(3)$ tensor objects. We define 


$$
\xi^{i j}=-\delta \chi^{0 i 0 j}, \quad \gamma^{i j}=\frac{1}{2} \epsilon^{j l m} \delta \chi^{0 i l m}, \quad \text { and } \zeta^{i j}=\frac{1}{4} \epsilon^{i l m} \epsilon^{j p q} \delta \chi^{l m p q},
$$

where $\epsilon^{i j k}$ is the Levi-Civita antisymmetric symbol. This decomposition is covariant under rotations of the quasi-Lorentzian coordinate system in which $\delta \chi^{\alpha \beta \gamma \delta}$ is represented. It follows that the tensors $\xi^{i j}, \gamma^{i j}$ and $\zeta^{i j}$ represent spatial anisotropy induced by the background gravitational field in a natural way. The symmetries of $\chi^{\alpha \beta \gamma \delta}$ and $\delta \chi^{\alpha \beta \gamma \delta}$ imply that $\xi^{i j}$ and $\zeta^{i j}$ are symmetric. Their twelve independent components combine with the nine of $\gamma^{i j}$ to account for the twenty-one nonmetric degrees of freedom of the $\chi g$ formalism. An analogous decomposition of $\chi^{\alpha \beta \gamma \delta}$ yields tensors $\epsilon^{i j}=\delta^{i j}+\xi^{i j}, \chi^{i j}=\delta^{i j}+\zeta^{i j}$ and $\gamma^{i j}$.

Consider the propagation of light in the neighborhood of some event in spacetime. We exploit the covariance of our decomposition of $\delta \chi^{\alpha \beta \gamma \delta}$ to rotate from the original quasiLorentzian $(t, x, y, z)$ coordinate system in which the background gravitational field is represented to a set of $\left(t, x^{\prime}, y^{\prime}, z^{\prime}\right)$ coordinates in which the light propagates in the $z^{\prime}$ direction. In this new coordinate system $\mathbf{A}_{B}$ has only $x^{\prime}$ and $y^{\prime}$ components so Eq. (15) reduces to the system of two equations,

$$
\begin{aligned}
& \left(1-\frac{\omega^{2}}{k^{2}}\right) A_{B}^{1^{\prime}}=\mathcal{A} A_{B}^{1^{\prime}}-\mathcal{B} A_{B}^{2^{\prime}}, \\
& \left(1-\frac{\omega^{2}}{k^{2}}\right) A_{B}^{2^{\prime}}=-\mathcal{B} A_{B}^{1^{\prime}}+\mathcal{C} A_{B}^{2^{\prime}} .
\end{aligned}
$$

The coefficients $\mathcal{A}, \mathcal{B}$ and $\mathcal{C}$ depend on location in spacetime and on the direction in which the wave propagates, the $z^{\prime}$ direction. This dependence is implicit in the expressions for $\mathcal{A}$, $\mathcal{B}$ and $\mathcal{C}$ in terms of the values at the event in question of the tensor components $\xi^{i^{\prime} j^{\prime}}, \zeta^{i^{\prime} j^{\prime}}$ and $\gamma^{i^{\prime} j^{\prime}}$ in the $\left(t, x^{\prime}, y^{\prime}, z^{\prime}\right)$ coordinate system,

$$
\begin{gathered}
\mathcal{A}=\xi^{2^{\prime} 2^{\prime}}-2 \gamma^{2^{\prime} 1^{\prime}}-\zeta^{1^{\prime} 1^{\prime}}, \\
\mathcal{B}=\xi^{1^{\prime} 2^{\prime}}+\left(\gamma^{2^{\prime} 2^{\prime}}-\gamma^{1^{\prime} 1^{\prime}}\right)+\zeta^{1^{\prime} 2^{\prime}},
\end{gathered}
$$

and 


$$
\mathcal{C}=\xi^{1^{\prime} 1^{\prime}}+2 \gamma^{1^{\prime 2^{\prime}}}-\zeta^{2^{\prime} 2^{\prime}}
$$

The matrix defining the structure of the right-hand sides of Eqs. (17) and (18) is real-valued and symmetric when $\delta \chi^{\alpha \beta \gamma \delta}$ is real. Its eigenvalues are

$$
\lambda_{ \pm}=\frac{\mathcal{A}+\mathcal{C}}{2} \pm \frac{1}{2} \sqrt{(\mathcal{A}-\mathcal{C})^{2}+4 \mathcal{B}^{2}}
$$

The corresponding eigenvectors define the polarization states that propagate with welldefined coordinate phase velocities $c_{ \pm}=1-\frac{1}{2} \lambda_{ \pm}+O\left(\delta \chi^{2}\right)$.

The effects of a nonmetric background field on physical standards of length and time are such that a local observer who uses rulers and clocks to measure velocities of light having the polarizations singled out by the field will generally obtain results that differ from the coordinate velocities $c_{+}$and $c_{-}$at $O(\delta \chi)$. To that order, however, the fractional difference between the velocities that an observer at rest in the $\left(t, x^{\prime}, y^{\prime}, z^{\prime}\right)$ coordinate system does measure will be the same as the fractional difference between $c_{+}$and $c_{-}$. We let $\delta c / c$ denote the magnitude of this dimensionless local observable,

$$
\frac{\delta c}{c}=\frac{1}{2} \sqrt{(\mathcal{A}-\mathcal{C})^{2}+4 \mathcal{B}^{2}}+O\left(\delta \chi^{2}\right)
$$

A practical way to measure or to impose an upper limit on this magnitude is to search for the effect such birefringence has on the polarization of light that propagates through the background gravitational field.

We use Stokes parameters based on the linear polarization states singled out by the background field to characterize the polarization of light that propagates through a local observer's laboratory. The intensity $I$ and the degrees of polarization $Q / I, U / I$ and $V / I$ provide a particularly convenient representation of the light's state. We may think of the light as an incoherent superposition of appropriate intensities of unpolarized light, linearly polarized light with $Q / I= \pm 1$ and elliptically polarized light with $\left(U^{2}+V^{2}\right)^{1 / 2} / I= \pm 1$. Propagation through the observer's laboratory only affects the state of the last of these components. 
The unpolarized component is itself an incoherent superposition of equal amplitudes of the basis polarization states while the linearly polarized component consists purely of one or the other of the basis polarization states. The shift in the relative phase of waves in the basis states that accumulates as these waves propagate with different phase velocity has no effect on the polarization of either of these components. On the other hand, the elliptically polarized component is a coherent superposition of equal amplitudes of the basis states so its polarization is sensitive to the relative phase of these waves. Indeed, the relative phase determines this component's $U / I$ and $V / I$ degrees of polarization, $V / U=\tan \left(\Phi_{+}-\Phi_{-}\right)$. As light propagates during a coordinate time interval $d t$ this relative phase shifts by $\omega(\delta c / c) d t$, where $\omega$ is the light's angular frequency. It is this shift that can affect light's polarization.

We piece together the cumulative effect of propagation through a background gravitational field on a signal's polarization from such local effects. This is particularly easy to do when the orientation of the basis polarization states singled out by the background field is fixed along light rays, as is the case for light propagating through the essentially static, spherically symmetric background fields that we consider in the next section. A ray threading through a spherically symmetric field lies in a plane and, for the fields we consider, there is a fixed orientation between this plane and the $\mathbf{A}_{B}$ amplitudes of the polarization states singled out by the fields. In such cases the relative phase shift that accumulates between waves in the basis states is simply

$$
\Delta \Phi=\omega \int \frac{\delta c}{c} d t+O\left(\delta \chi^{2}\right),
$$

where, since the local value of $\delta c / c$ is explicitly $O(\delta \chi)$, the integration runs along the unperturbed ray that runs straight through the quasi-Lorentzian coordinate system with unit speed from source to receiver. The integrand in Eq. (24) is the local value of $\delta c / c$ for the ray's direction.

One consequence of the relative phase shift (24) is that a signal whose elliptically polarized component has $V / U=0$ when emitted will have some degree of circular polarization when it is received. The degree of circular polarization received is proportional to a small 
accumulated shift,

$$
V / U \approx \Delta \Phi
$$

We base our interpretation of galaxy polarization data in the next section on this expression.

We have seen that a nonmetric background field induces birefringence if and only if $(\mathcal{A}-\mathcal{C})$ or $\mathcal{B}$ in Eq. (23) is nonzero for some direction of wave propagation at some event in spacetime. Before we turn to the derivation of empirical limits on such birefringence let us consider how such limits constrain the form of the coupling between gravitational and electromagnetic fields.

Note that the expressions $(\mathcal{A}-\mathcal{C})$ and $\mathcal{B}$ involve only the symmetric, trace-free parts of the tensors $\xi^{i j}, \zeta^{i j}$ and $\gamma^{i j}$. This is most easily appreciated by writing $(\mathcal{A}-\mathcal{C})$ and $\mathcal{B}$ in terms of the spherical components of these tensors. In terms of components in the $\left(t, x^{\prime}, y^{\prime}, z^{\prime}\right)$ coordinate system they are

$$
\mathcal{A}-\mathcal{C}=\frac{2}{\sqrt{6}}\left[\left(\xi_{2^{\prime}}^{(2)}+\xi_{-2^{\prime}}^{(2)}\right)+2 i\left(\gamma_{2^{\prime}}^{(2)}-\gamma_{-2^{\prime}}^{(2)}\right)+\left(\zeta_{2^{\prime}}^{(2)}+\zeta_{-2^{\prime}}^{(2)}\right]\right.
$$

and

$$
\mathcal{B}=-\frac{1}{\sqrt{6}}\left[i\left(\xi_{2^{\prime}}^{(2)}-\xi_{-2^{\prime}}^{(2)}\right)+2\left(\gamma_{2^{\prime}}^{(2)}+\gamma_{-2^{\prime}}^{(2)}\right)+i\left(\zeta_{2^{\prime}}^{(2)}-\zeta_{-2^{\prime}}^{(2)}\right)\right]
$$

Only $l=2$ components appear. Expressions for $(\mathcal{A}-\mathcal{C})$ and $\mathcal{B}$ in terms of components in the original $(t, x, y, z)$ coordinate system follow from the transformation law for spherical tensor components [10], for example,

$$
\xi_{m^{\prime}}^{(l)}=\mathcal{D}_{m^{\prime} m}^{(l)}(\phi, \theta, \psi) \xi_{m}^{(l)}
$$

where $\phi, \theta$ and $\psi$ are the Euler angles specifying the rotation from $(t, x, y, z)$ to $\left(t, x^{\prime}, y^{\prime}, z^{\prime}\right)$. From the form of this transformation law and of Eqs. (26) and (27) we conclude that the absence of gravity-induced birefringence implies $\xi_{m}^{(2)}+\zeta_{m}^{(2)}=0$ and $\gamma_{m}^{(2)}=0$ and, so, that observations imposing limits on the strength of gravity-induced birefringence constrain ten of the $\chi g$ formalism's twenty-one nonmetric degrees of freedom. This conclusion and the expression (23) for $\delta c / c$ are in accord with remarks of $\mathrm{Ni}[7]$ for which the preceding analysis provides a detailed justification. 


\section{GRAVITY-INDUCED BIREFRINGENCE AND THE POLARIZATION OF GALAXIES}

The preceding analysis examined nonmetric couplings between the gravitational and electromagnetic fields and established which single out pairs of linear polarization states that propagate through a gravitational field with different phase velocities. Using Stokes parameters based on those states, we found that such gravity-induced birefringence causes $U$-polarized radiation to develop a degree of circular polarization as it propagates.

Here, we interpret polarization measurements of extragalactic sources cataloged by Weiler and De Pater [11] in light of this prediction and derive a precise new constraint on the strength of birefringence that could be induced by the Galaxy's gravitational field. Note that even a cursory examination of the data indicates that such birefringence cannot be strong. If it were, one would expect to measure substantial circular and linear polarization of radiation from extragalactic sources with about the same likelihood. This is not the case. Measurements reveal significant circular polarization of radiation from extragalactic sources only rarely while they frequently reveal substantial linear polarization.

As a first approximation, we treat the Galaxy's gravitational field as static and spherically symmetric. This is not unreasonable, especially if one accepts the existence of a substantial dark-matter halo. Note that the assumed spherical symmetry does not preclude the detection

of gravity-induced birefringence. Radiation reaching the Earth from an extragalactic source generally does not propagate radially through the Galaxy's field because the Earth lies 10 kiloparsecs from the Galaxy's center. A ray along which such radiation travels lies in a plane defined by the locations of the source, the Earth and the center of the Galaxy.

At each point along a ray the only direction that the Galaxy's spherical field can single out is the radial one. We, therefore, find it useful to introduce at each of these points a local quasi-Lorentzian $(t, x, y, z)$ coordinate system oriented so that its $z$ axis is radial and its $x$ axis lies in the ray's plane. We do so because the representation of the spherical tensors introduced in the preceding section is simple in these local systems. Specifically, $\xi_{m}^{(2)}, \zeta_{m}^{(2)}$ 
and $\gamma_{m}^{(2)}$ are nonzero only for $m=0$. The nonzero components depend only on the radial coordinate of the point along the ray.

At each of these points we can rotate the local $(t, x, y, z)$ coordinate system about its $y$ axis through an angle $\theta$ to obtain a local $\left(t, x^{\prime}, y^{\prime}, z^{\prime}\right)$ coordinate system in which the ray runs in the $z^{\prime}$ direction. Equation (23) expresses the local value of $\delta c / c$ for points on the ray in terms of $(\mathcal{A}-\mathcal{C})$ and $\mathcal{B}$ which are, in turn, expressed in terms of the local $\left(t, x^{\prime}, y^{\prime}, z^{\prime}\right)$ components $\xi_{ \pm 2^{\prime}}^{(2)}, \zeta_{ \pm 2^{\prime}}^{(2)}$ and $\gamma_{ \pm 2^{\prime}}^{(2)}$ by Eqs. (26) and (27). Since the Euler angles of the rotation from $(t, x, y, z)$ to $\left(t, x^{\prime}, y^{\prime}, z^{\prime}\right)$ are $\phi=0, \theta$ and $\psi=0$, the transformation law (28) implies

$$
\xi_{ \pm 2^{\prime}}^{(2)}=\sin ^{2}(\theta) \xi_{0}^{(2)}
$$

with the same relationship holding between $\zeta_{ \pm 2^{\prime}}^{(2)}$ and $\zeta_{0}^{(2)}$ and between $\gamma_{ \pm 2^{\prime}}^{(2)}$ and $\gamma_{0}^{(2)}$. For any spherically symmetric gravitational field, we conclude that $\delta c / c$ is proportional to the square of the sine of the angle by which a ray's direction differs from the radial one.

The orientations of the $\mathbf{A}_{B}$ amplitudes of the polarization states singled out along a ray are determined by the relative magnitudes of the $(t, x, y, z)$ components $\xi_{0}^{(2)}, \zeta_{0}^{(2)}$ and $\gamma_{0}^{(2)}$. The form of Eqs. (26) and (27) and the transformation law (29) imply that $\mathcal{B}$ is proportional to $\gamma_{0}^{(2)}$ while $(\mathcal{A}-\mathcal{C})$ is proportional to $\left(\xi_{0}^{(2)}+\zeta_{0}^{(2)}\right)$. In the event that $(\mathcal{A}-\mathcal{C}) \neq 0$ and $\mathcal{B}=0$, Eqs. (17) and (18) imply that for the polarization states singled out by the Galaxy's field $\mathbf{A}_{B}$ will lie in and perpendicular to the ray's plane. On the other hand, when $(\mathcal{A}-\mathcal{C})=0$ and $\mathcal{B} \neq 0$, Eqs. (17) and (18) imply the $\mathbf{A}_{B}$ singled out will be oriented at 45 degrees to either side of the normal to the ray's plane. Cases like these, in which the polarization states singled out by a background field are fixed along rays, are special. Generally, their orientations will change along a ray as the relative magnitudes of $\xi_{0}^{(2)}, \zeta_{0}^{(2)}$ and $\gamma_{0}^{(2)}$ vary.

The effect that propagation through the Galaxy's gravitational field has on the polarization of light from extragalactic sources clearly depends on the global structure of that gravitational field. We choose to compute the effect for an NGT model of the Galaxy's field studied by Krisher [4] so that we can compare the precision of the constraint we impose on gravity-induced birefringence with the one he imposed. This NGT field has $\xi_{0}^{(2)}=0$, 
$\zeta_{0}^{(2)}=L^{2}(r)$ and $\gamma_{0}^{(2)}=0$, where the function $L^{2}(r)$ specifies the structure of the antisymmetric tensor part of the Galaxy's nonsymmetric field. The fact that only $\zeta_{0}^{(2)}$ is nonzero is a consequence of the nonmetric coupling between the nonsymmetric field and the electromagnetic field having been tuned to satisfy constraints imposed by other tests of the Einstein equivalence principle [12]. Since only $\zeta_{0}^{(2)}$ is nonzero, the orientations of the basis polarization states singled out by the Galactic field are fixed along rays with their magnetic amplitudes lying in and perpendicular to ray planes. Consequently, Eq. (24) gives the relative phase shift that accumulates between waves in these polarization states as they propagate. We have $\delta c / c=\frac{1}{2} L^{2} \sin ^{2}(\theta)$, which is consistent with the results of Gabriel [1,3] et al. since they denote $L^{2}$ by $\Omega$.

In Krisher's model the antisymmetric part of the Galaxy's nonsymmetric gravitational field is generated by a uniform density, spherical halo of weakly interacting particles. Its radius, $R$, is 25 kiloparsecs. The function $L^{2}(r)$ is proportional to the halo's net NGT charge, $l^{2}$. We have $L^{2}=l^{4} / r^{4}$ for radii greater than $R$ and $L^{2}=l^{4} r^{2} / R^{6}$ for smaller radii. The NGT charge, $l^{2}$, can be expressed in terms of a coupling constant $f_{c}^{2}$ and the density of halo particles, $n_{c}$, which Krisher takes to be 0.1 per cubic centimeter. Specifically, $l^{2}=\left(4 \pi R^{3} / 3\right) n_{c} f_{c}^{2}$.

The geometry of a ray that runs from an extragalactic source to the Earth is shown in Fig. 1. The spherical symmetry of the Galaxy's field implies an axial symmetry of the relative phase shift (24). It depends only on the angle $\beta$. The 10 kiloparsec distance between the Earth and the Galaxy's center is denoted by $d$. The ray's impact parameter is $b=d \sin (\beta)$. To compute the phase shift (24), we parametrize the rays so that the point $r=b$, which may lie on an extension of the ray, corresponds to $t=0$ and we suppose that the ray starts from $r=\infty$ at $t=-\infty$. It follows that the ray encounters the halo's surface at $t=t_{R} \equiv-\left(R^{2}-b^{2}\right)^{1 / 2}$ and the Earth at $t=t_{E} \equiv-d \cos (\beta)$. Note that $r^{2}=\left(b^{2}+t^{2}\right)$ and $\sin ^{2}(\theta)=b^{2} /\left(b^{2}+t^{2}\right)$ along the ray.

From these facts and the expressions for $L^{2}$ inside and outside the halo we conclude that 


$$
\Delta \Phi_{e x t}=\frac{\omega l^{4}}{2} \int_{-\infty}^{-t_{R}} \frac{b^{2} d t}{\left(b^{2}+t^{2}\right)^{3}}=\left.\frac{\omega l^{4}}{8}\left(\frac{t}{\left(b^{2}+t^{2}\right)^{2}}+\frac{3 t}{2 b^{2}\left(b^{2}+t^{2}\right)}+\frac{3}{2 b^{3}} \arctan \left(\frac{t}{b}\right)\right)\right|_{-\infty} ^{t_{R}}
$$

and

$$
\Delta \Phi_{i n t}=\frac{\omega l^{4} b^{2}}{2 R^{6}} \int_{t_{R}}^{t_{E}} d t=\frac{\omega l^{4} b^{2}}{2 R^{6}}\left(t_{E}-t_{R}\right) .
$$

Equation (30) corrects a misprint in Krisher's expression for $\Delta \Phi_{\text {ext }}$. More seriously, Krisher's numerical estimate of $\Delta \Phi_{\text {ext }}$ indicates that $t_{E}$ rather than $t_{R}$ was used as an upper limit of integration. This contribution to $\Delta \Phi$ was, therefore, significantly overestimated. In Fig. 2 we plot conveniently normalized functions $F_{\text {ext }}(\beta)$ and $F_{\text {int }}(\beta)$ defined by $\Delta \Phi_{i}=\left(\omega l^{4} / d^{3}\right) F_{i}$.

Data from the catalog of Weiler and De Pater impose a sharp new limit on the amplitude of $\Delta \Phi(\beta)$ and, so, on the magnitudes of $l^{2}$ and $f_{c}^{2}$.

From the position of a source in the sky and a bit of geometry we obtain both the angle $\beta$ for the source and the orientation of the polarization states singled out for it by the Galaxy's field. We use this latter information to express the source's cataloged polarization in terms of Stokes parameters based on the polarization states singled out. Since the relative phase shift $\Delta \Phi$ converts $U$ polarized light to $V$ polarized light, we calculate $V / U$ for sources with accurately measured $U / I$ and $V / I$ and for which the $U / I$ degree of polarization is substantial, accounting for more than half of the observed linear polarization. The substantial $U / I$ degree of polarization means that a given relative phase shift will induce a relatively large $V / I$ and it also assures that $V / U$ will be a well-behaved indicator of $\Delta \Phi$ via Eq. (25) where we make the conservative assumption that a nonzero average of $V / U$ for sources in an interval around $\beta$ represents the effect of the phase shift $\Delta \Phi(\beta)$. Figure 3 is a plot of the $V / U$ as a function of $\beta$ for data taken at $5 \mathrm{GHz}$, the highest frequency included in the Weiler-De Pater catalog. The average of $V / U$ for these sources is consistent with zero. We estimate limits imposed by this data by noting that 0.12 is the sample standard deviation of $V / U$ for the $5 \mathrm{GHz}$ data having $\beta>60$ degrees. Equation (25) imposes this as a limit on the mean of $\Delta \Phi$ for $\beta$ in this range. This limit and the expression in terms of $f_{c}^{2}$ for the mean of $\Delta \Phi(\beta)$ in the range from 60 to 90 degrees implies $f_{c}^{2}<1.4 \times 10^{-34} \mathrm{~cm}^{2}$. When the means and sample standard 
deviations of $V / U$ for lower frequency data are accounted for this constraint is sharpened slightly,

$$
f_{c}^{2}<1.3 \times 10^{-34} \mathrm{~cm}^{2}
$$

This limit is more than $10^{4}$ times tighter than the one Krisher derives, once his overestimate of the exterior contribution to $\Delta \Phi$ is corrected for. This increase in precision is easily understood. Krisher argued that NGT-induced birefringence would affect the polarization structure of pulses from pulsars in the Large and Small Magellenic Clouds and reckoned that an effect would have been noticed if the arrival times of pulse components in the polarization states singled out by the Galaxy's field were shifted relative to each other by as much as a millisecond. In our case, we use a kind of interferometry to discern a much smaller relative time delay. The highest frequency data that we analyze imposes a limit of 0.12 radians on the relative phase shift between the polarization states singled out by the Galaxy's field. We are, therefore, resolving a fraction $0.12 / 2 \pi$ of a cycle at the $5 \mathrm{GHz}$ observation frequency. This corresponds to a relative time delay of $4 \times 10^{-12}$ seconds. Our time resolution is, therefore, more than $10^{8}$ times Krisher's. Since, as Krisher remarks, constraints on the magnitudes of $l^{2}$ or $f_{c}^{2}$ are proportional to the square root of the time interval resolved, the precision of our constraint (32) is accounted for.

\section{SUMMARY AND CONCLUSIONS}

The local isotropy of space is one consequence of the Einstein equivalence principle. Atomic physics experiments of Hughes-Drever [5] type are the classic tests of this predic-

tion. In this paper we have shown that searching for effects of propagation through a gravitational field on the polarization of light provides a precise, qualitatively different test for spatial anisotropy, specifically spatial anisotropy that induces a birefringence of space. In the context of the $\chi g$ formalism such anisotropy is associated with ten of twenty-one nonmetric degrees of freedom. 
The significance of any test of the Einstein equivalence principle lies in the constraint it imposes on the form of the coupling between gravitational and matter fields. Constraints on gravity-induced birefringence force the structure of the electromagnetic Lagrangian density (3) towards the form having

$$
\chi^{\alpha \beta \gamma \delta}=\frac{1}{2} \sqrt{-\tilde{g}}\left(\tilde{g}^{\alpha \gamma} \tilde{g}^{\beta \delta}-\tilde{g}^{\alpha \delta} \tilde{g}^{\beta \gamma}\right)+\phi \epsilon^{\alpha \beta \gamma \delta} .
$$

That the phenomenological symmetric tensor potential $\tilde{g}^{\alpha \beta}$ and scalar potential $\phi$ can represent the $\chi g$ formalism's remaining eleven nonmetric degrees of freedom is clear upon counting components. The fact that this Lagrangian density predicts no birefringence in the geometric optics limit is clear from the metric form of the coupling between $\tilde{g}^{\alpha \beta}$ and the electromagnetic field and from the identification of $\phi$ as the $\gamma_{0}^{(0)}$ part of $\delta \chi^{\alpha \beta \gamma \delta}$. Note, however, that Carroll and Field [13] have shown that at post-geometric-optics order the $\phi$ coupling does induce a dispersive optical activity which they have constrained using galaxy polarization data.

Two features are primarily responsible for the precision of Einstein equivalence principle tests based on the interpretation of polarization data for extragalactic sources. First, they focus on global consequences of Einstein equivalence principle violations. They constrain shifts in relative phase that accumulate from minute local effects rather than seeking the local effects directly. Second, they are interferometric in character. Their sensitivity to accumulated phase shifts comes from exploiting the coherence inherent in polarized light. Note that the sharp new constraint on NGT derived above is far beyond the reach of local Hughes-Drever experiments, as indicated by an estimate analogous to one that Gabriel [1] et al. make of local anisotropy induced by the Sun's field in NGT.

Sharper tests of the Einstein equivalence principle are all but certain to result from surveys of extragalactic sources that yield linear and circular polarization measurements at frequencies higher than the $5 \mathrm{GHz}$ limit of the Weiler-De Pater catalog. 


\section{REFERENCES}

[1] M. D. Gabriel, M. P. Haugan, R. B. Mann and J. H. Palmer, Phys. Rev. D43, 308 (1991) and Phys. Rev. D43, 2465 (1991).

[2] J. W. Moffat in Gravitation: A Banff Summer Institute edited by R. B. Mann and P. Wesson (World Scientific, Singapore,1991) and references therein.

[3] M. D. Gabriel, M. P. Haugan, R. B. Mann and J. H. Palmer, Phys. Rev. Lett. 67, 2123 (1991).

[4] T. P. Krisher, Phys. Rev. D44, R2211 (1991).

[5] V. W. Hughes, H. G. Robinson and V. Beltran-Lopez, Phys. Rev. Lett. 4, 342 (1960); R. W. P. Drever, Phil. Mag. 6, 683 (1960); J. D. Prestage et al., Phys. Rev. Lett. 54, 2387 (1985); S. K. Lamoreaux et al., Phys. Rev. Lett. 57, 3125 (1986) and T. E. Chupp et al., Phys. Rev. Lett. 63, 1541 (1989).

[6] C. M. Will, Theory and Experiment in Gravitation Physics, (Cambridge University Press, Cambridge, England, 1981).

[7] W. - T. Ni in Precision Measurements and Fundamental Constants II, editied by B. N. Taylor and W. D. Phillips, U. S. National Bureau of Standards Special Publication No. 617 (U. S. GPO, Washington, D. C. , 1984).

[8] K. S. Thorne, D. L. Lee and A. P. Lightman, Phys. Rev. D7, 3563 (1973).

[9] W. - T. Ni, Phys. Rev. Lett. 38, 301 (1977).

[10] A. R. Edmonds, Angular Momentum in Quantum Mechanics, (Princeton University Press, Princeton 1974).

[11] K. W. Weiler and I. De Pater, Ap. J. Suppl. 52, 293 (1983).

[12] R. B. Mann, J. H. Palmer and J. W. Moffat, Phys. Rev. Lett. 62, 2765 (1989). 
[13] S. M. Carroll and G. B. Field, Phys. Rev. D43, 3789 (1991). 


\section{FIGURES}

FIG. 1. Points $G, E$, and $S$ depict the locations of the Galaxy's center, the Earth and an extragalactic source. A halo of radius $R=25 k p c$ surrounds the galaxy. The Earth lies at a distance $d=10 \mathrm{kpc}$ from $G$. Light from the source $S$ propagates to the Earth along the ray $S E$. The relative phase shift that accumulates depends only on the angle $\beta$ or, equivalently, on the impact parameter $b=d \sin (\beta)$. At each point $P$ of $S E$ we denote the angle between $S E$ and the radial line $G P$ by $\theta(P)$.

FIG. 2. The function of $\beta$ depicted by the solid curve is proportional to the relative phase delay. It is the sum of the internal and external contributions, $F_{\text {int }}(\beta)$ and $F_{\text {ext }}(\beta)$, defined in the text and denoted here by dotted and dashed curves respectively.

FIG. 3. Plot of $V / U$ versus $\beta$ for $5 \mathrm{GHz}$ data from the Weiler-De Pater catalog [11]. The apparent clustering of data is a consequence of including data taken at different times for some sources. 


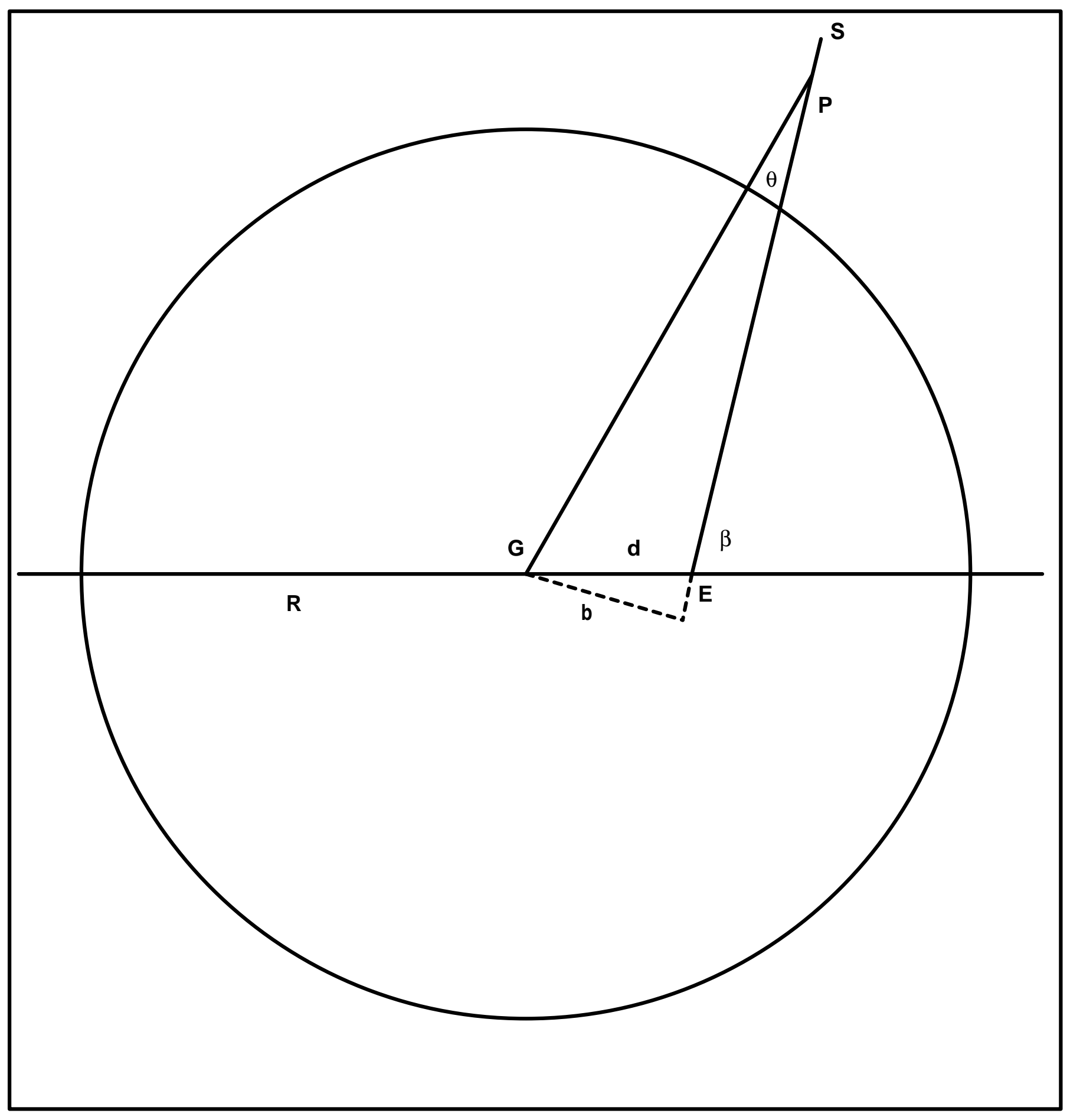




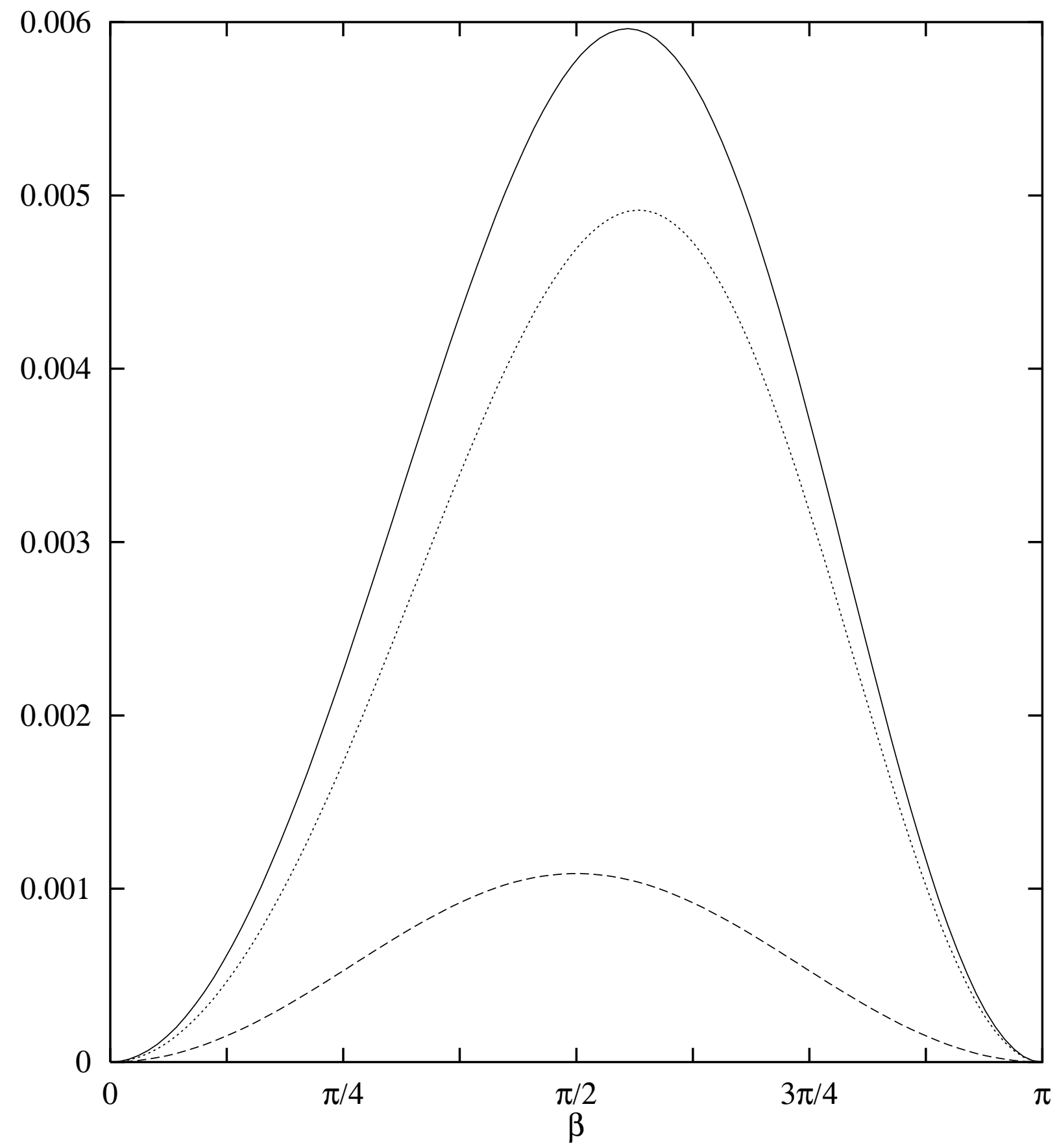




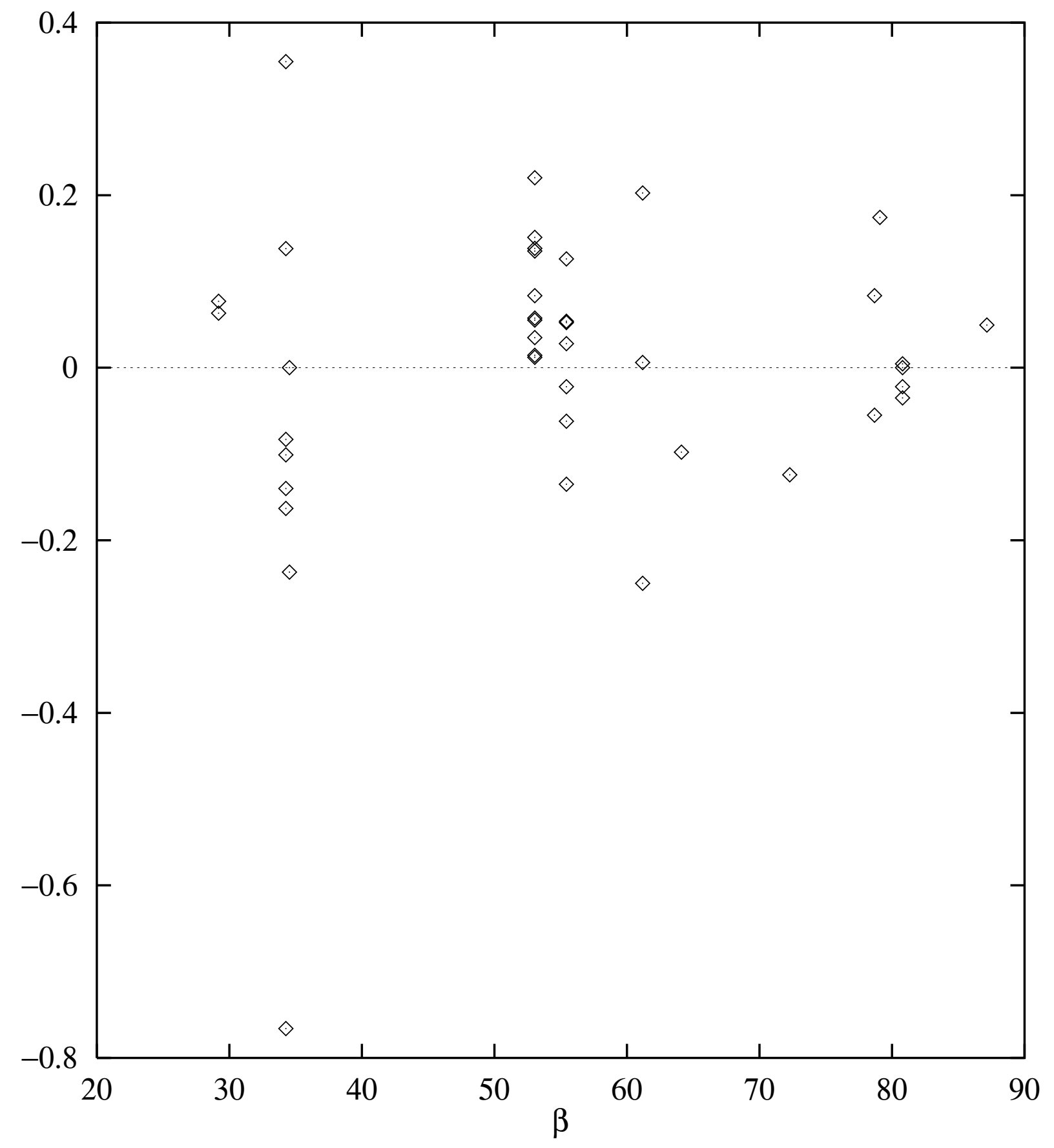

